\title{
An Analytical Model to Estimate Path Duration in MANETs
}

\author{
A. Triviño-Cabrera, J. García-de-la-Nava, E. Casilari, F.J. González-Cañete \\ Dpto. Tecnología Electrónica \\ Campus de Teatinos, \\ Málaga (SPAIN) \\ 34952137191 \\ atc@uma.es
}

\begin{abstract}
Mobile Ad Hoc Networks (MANETs) are strongly impacted by the mobility of the ad hoc nodes. Mobility models help engineers to abstract the changes of position in network users. Different mobility models have already been proposed to characterize the behaviors of mobile devices that are greatly correlated to the conditions of the possible scenarios where networks can be deployed. In the context of mobile ad hoc networks, the Random WayPoint model has received significant attention and it has become one of the most employed mobility patterns. However, a formal description of the time during which a generic $N$-hop path is valid has not been proposed yet for this model. In this paper, the authors provide an analytical model for link duration as well as an analytical study of path duration in multi-hop wireless networks. The predicted results are compared with measured data and good agreement is reported.
\end{abstract}

\section{Categories and Subject Descriptors}

C.2.2 [Network Protocols]: Routing Protocols

\section{General Terms}

Reliability, Theory

\section{Keywords}

MANET, Path Duration, Link Duration, Random WayPoint.

\section{INTRODUCTION}

Among the multiple scenarios where ubiquitous computing may be present, the Mobile Ad Hoc Networks are receiving significant attention. Mobile Ad hoc Networks (MANET) allow wireless devices to intercommunicate without the use of any pre-existing infrastructure. However, the absence of any centralized entity transfers the routing procedures to the nomadic ad hoc terminals as direct communication is only possible between neighbouring nodes. Thus, distant nodes require the collaboration of intermediate terminals that should forward the source packets to the final destination through multiple links or hops.

Permission to make digital or hard copies of all or part of this work for personal or classroom use is granted without fee provided that copies are not made or distributed for profit or commercial advantage and that copies bear this notice and the full citation on the first page. To copy otherwise, or republish, to post on servers or to redistribute to lists, requires prior specific permission and/or a fee.

MSWiM'06, October 2-6, 2006, Torremolinos, Malaga, Spain.

Copyright 2006 ACM 1-59593-477-4/06/0010 ...\$5.00.
The method for selecting the intermediate nodes as well as the response to any link break in the path are the main characteristics that differentiate the existing ad hoc routing protocols. These protocols can be roughly divided into three categories: proactive, reactive and hybrid. In proactive or table driven protocols nodes send messages periodically in order to update the topological information about the network, so that a route is immediately available as soon as a new connection is required. On the contrary, in reactive or on-demand protocols, nodes generate route queries in order to discover a needed route to a particular node. When a response is back-propagated by the network to the origin node, nodes cache the configured routes for a certain period of time. Finally, hybrid protocols combine both strategies as they follow proactive scheme for a restricted closer zone around the sending node while it employs the reactive technique to communicate with nodes out of this defined proactive area.

In general terms, routing protocols requires the configuration of some parameters which greatly influences on the network performance. Usually, the specifications recommend some default values for these parameters that do not follow any experimentally evaluated criterion, so that the values differ in various specifications. Specifically, in the case of reactive protocols, the time that a route is considered to be valid varies depending on the particular algorithm that is being utilized. For instance, in AODV with enabled link layer detection, stored routes become invalid after 10 seconds (the Active Route Timeout) without being utilized [1]. Meanwhile DSR and DYMO contemplate another time constant equal to 3 and 300 seconds (RouteCacheTimeout and RouteTimeout respectively) to suppress paths from the node caches [2] [3]. These time-out periods are applied for all the nodes without taking into consideration the mobility conditions or the route length (estimated by the number of hops). In order to select an appropriate value for this parameter as well as to know its main impacting factors, an analysis of the time that a path is valid should be performed. In this sense, the aim of this work is to present a statistical characterization of the duration of routes in mobile multi-hop wireless networks. This characterization can be of great help for the routing protocols to select stable routes or proper values for the route timeouts. Additionally, the prior assumption of the disruption of a route may be useful to initiate the route discovery procedures earlier, diminishing the delay associated to reactive protocols [4].

The rest of the paper is organized as follows. Section 2 presents a short survey on related work in the area. Link duration for the Random WayPoint is studied in Section 3. Section 4 explains the analytical model for the path duration. This model is verified by 
extensive simulations described in section 5. Finally, Section 6 draws the main conclusions of the paper.

\section{RELATED WORK}

Simulation has been the main method for analyzing the properties of route or path duration in ad hoc networks. One of the first studies concerning the analysis of path duration was due to Bai et al. [5]. Basing on experimental results obtained by simulations, they assume that the lifetime of a path with four or more hops can be approximated by an exponential distribution. However the authors do not consider the fit of any other standard distribution. Moreover, they do not justify the selection of an exponential distribution with any mathematical validation. To cope with this lack, Han et al., basing their work on Palm's theorem, state that, under some circumstances, the lifetime associated to those paths with a large number of hops converges to an exponential distribution [6]. The previous works present a clear disadvantage as they provide a solution for the analysis of paths which is valid only for routes with a large number of hops. Therefore their study could not be fully applied to usual ad hoc networks and practical MANET applications where the paths only consist of 1 to 4 hops [7]. The importance of short paths is reinforced by the fact that the most relevant protocols utilize the minimum hop count as the metric to select the route in use in order to reduce the effects of the wireless retransmissions on the performance of the network. Anyway, the popularity of the exponential fitting has made it a common approximation in some other works as in [8] [9].

Most authors have analyzed path duration by means of empirical results. For instance, [10] have shown that the mean residual lifetime of routes depends on the number of hops as well as on the mean Link Duration. On the other hand, [4] analytically proves that the average lifetime of a path decreases with its length.

An analytical study on this issue is due to Tseng et al. They base the analysis of the route lifetime on a spatial discrete model [11]. This study simplifies a MANET into a cellular network composed by hexagonal cells to compute the path availability. In [12], authors formally describe the distribution function of path duration assuming that nodes move according to a constant velocity model.

In this paper, we develop an analytical framework in order to analyze the factors on the lifetime of a path that is formed by an arbitrary number of hops.

\section{LINK DURATION}

There exist several mobility patterns that try to capture the behavior of the mobile devices under different circumstances. In this sense, entity and group mobility models have been proposed [13]. In the entity mobility pattern, movements of nodes are independent of the movements of the rest of the nodes that belongs to the same network. On the other hand, in the group mobility models, the movements of different nodes are correlated.

Although link duration is considered a fundamental parameter when evaluating the mobility in a MANET [14], few studies analyze a formal description of this variable. Some authors have formally studied the link duration for specific mobility models. For instance, [10] have studied the mean value of link duration under a constant velocity model meanwhile [4] analyzed it for a deterministic, partially deterministic and Brownian mobility model. On the other hand, [15] formally described the statistics associated to the link duration for a simplified random mobility model. In [16], a thorough analytical study of some significant parameters of link duration is presented. The main disadvantage is that it is only based on the Constant Velocity Model.

One of the most extended individual mobility models is the Random WayPoint (RWP) whose generic formulation was considered unreliable. In order to suppress the instability in the results, authors in [17] propose the incorporation of a simple modification that consists of fixing a minimum non zero speed in the nodes. This mobility pattern is called the Modified Random WayPoint. Due to its popularity as well as its capability of characterize multiple scenarios, the authors of this contribution will continue the analysis of link and path duration assuming nodes follow the Modified Random WayPoint Model. Despite the Modified RWP is widely utilized as a mobility pattern, at the present, no analytical formulation has related the statistical properties of the link duration to the parameters of the modified RWP, the network dimensions, the node density or the transmission range. The only found references are based on simulations [18]. In general, the authors suggest the approximation to a standard distribution function without any mathematical support [5] [19].

In this section, we approach the link duration fitting when nodes follow the Modified RWP. The following standard distributions are utilized: Normal, Gamma, Weibull, Rayleigh, Pareto, Exponential and Lognormal. The parametrization of the approximations are based on the Maximum LikeHood Estimation (MLE) Adjustment. For this purpose, we evaluate the fittings by means of the Kolmogorov-Smirnof goodness-of-fit test (K-S test). The K-S test is an extended tool that captures the maximum deviation of the hypothesized Cumulative Distribution Function respect to the data to approximate.

The computation of link duration is obtained by the utilization of a developed module that was incorporated into the Matlab tool [20]. The basis of this module resides in the computation of the connectivity graph. A connectivity graph informs about the nodes that are neighbors, that is, that are directly connected because the distance that separates them is lower than the transmission range. Differences in the evolution of the connectivity graph imply the break or the creation of links.

In order to support the results obtained by the developed algorithm, the distribution of link duration is also computed from traces obtained when the NS-2 Simulator tool is employed [21]. For this purpose, the AODV routing protocol was utilized emitting periodic Hello messages [1].

To simulate the conditions on which multi-hop wireless networks can be deployed, we have evaluated the link duration varying the simulation area $\left(1500 \times 300 \mathrm{~m}^{2}\right.$ and $\left.2000 \times 2000 \mathrm{~m}^{2}\right)$, the maximum speed $(5,10,15 \mathrm{~m} / \mathrm{s})$, the pause time $(0,50 \mathrm{~s})$, the node density $(15,50$ mobile nodes) and the transmission range $(100,250 \mathrm{~m})$ in 10000 -second simulations.

For an easier understanding, the results of the K-S tests are averaged and included in Figure 1 where the straight lines represent the standard deviation. As it can be observed, the best approximation is obtained by a lognormal distribution function. It must be noted that for each of the performed simulations, the lognormal distribution is always the best fitting according to the $\mathrm{K}-\mathrm{S}$ test. 


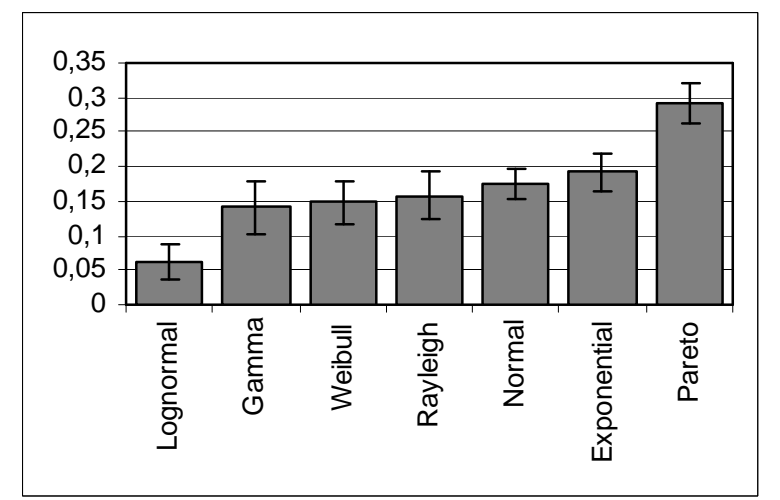

Figure 1. Mean and deviation results of the K-S tests over 50 scenarios

From the results of Figure 1, we can conclude that the lognormal distribution provides a better fitting to link duration although the Exponential and the Rayleigh functions have been widely utilized as the best approximation [5],[19].

\section{PATH DURATION}

In multi-hop wireless networks, a path is a sequence of communication links that are formed by adjacent neighbors. Due to node mobility, these links are broken and thus, the path lifetime becomes finite. This lifetime, called path duration, represents the elapsed time from the generation of the path to its break.

\subsection{Description of Path Duration}

Since a path between two nodes becomes invalid as soon as one of its links is broken, the path duration is equal to the minimum of the residual life of the links that conforms that route. Consequently, the distribution function of route duration $(R)$ can be mathematically expressed as:

$$
P(R \leq t)=P\left(\min _{i=1}^{N} L_{i} F_{i} \leq t\right)
$$

where $F_{i}$ represents the fraction of lifetime still remaining when the path is created meanwhile $L_{i}$ is the duration of the $i$-th link in an $N$-hop path, i.e. $1 \leq i \leq N$. It is necessary to include the $F_{i}$ factor as each link may have already been active an interval of time before the path was discovered. We suppose that the lifetimes of different links in the path could be considered mutually independent except for the case of adjacent links as the movement of a node may simultaneously affect the duration of two adjacent links. However, we will assume that the effect of the correlation of the duration for adjacent links is negligible as it has been reported in [6]. Under this assumption and considering that the distribution for the duration is the same for all the links, the previous expression can be further simplified into:

$$
P(R \leq t)=1-\prod_{i=1}^{N} P\left(L_{i} F_{i}>t\right)=1-(P(L \cdot F>t))^{N}
$$

$F_{i}$ and the link duration $L_{i}$ are independent variables, so:

$$
P(R \leq t)=1-\left(\int_{0 t / x}^{1} \int_{t}^{\infty} f_{F}(x) \cdot f_{L}(y) d y d x\right)^{N}
$$

$$
P(R \leq t)=1-\left(\int_{0}^{1} f_{F}(x) \int_{t / x}^{\infty} f_{L}(y) d y d x\right)^{N}
$$

where $f_{F}(x)$ is the probability density function of the fraction of use while $f_{L}(y)$ is the probability density function of the link duration. We assume that the factor of use can be modeled as a uniformly distributed random variable in the [0-1] interval (which implies $\left.f_{F}(x)=1\right)$. So the above equation is further simplified into:

$$
P(R \leq t)=1-\left(\int_{0 t / x}^{1} \int_{L}^{\infty} f_{L}(y) d y d x\right)^{N}
$$

\subsection{Application to the Modified RWP}

Although Equation 5 is valid independently on the mobility pattern that the nodes follow, the distribution function of the link duration $\left(f_{\mathrm{L}}\right)$ depends on the mobility characteristics. The Modified Random Waypoint is not associated to any closed expression that relates statistical properties of the link duration to the parameters of the RWP. However, the experimental results reflect a lognormal behavior as shown in section 3. Therefore, substituting in Equation 5 the $f_{L}$ as a lognormal function of parameters $\sigma$ and $\mu$, we have:

$$
P(R \leq t)=1-\left(\int_{0 t / x}^{1} \int_{x}^{\infty} \frac{1}{y \sigma \sqrt{2 \pi}} \exp \left(-\frac{1}{2}\left(\frac{\log (y / \mu}{\sigma}\right)^{2}\right) d y d x\right)^{N}
$$

The resolution of this integral yields to:

$$
P(R \leq t)=1-\left(\frac{1}{2} \int_{0}^{1} \operatorname{erfc}\left[\frac{\log (t / x)-\log (\mu)}{\sigma \sqrt{2}}\right] d x\right)^{N}
$$

from which we can obtain that:

$$
\left.P(R \leq t)=1-\left(\begin{array}{l}
\frac{1}{2} \operatorname{erfc}\left(\frac{\log (t / \mu)}{\sigma \sqrt{2}}\right)+\ldots \\
\ldots+\frac{t}{2 \mu} \cdot \exp \left(\frac{\sigma^{2}}{2}\right) \cdot \operatorname{erfc}\left(\frac{\log (t / \mu)-\sigma^{2}}{\sqrt{2} \sigma}\right)
\end{array}\right)\right)_{(8)}^{N}
$$

Therefore, Equation 8 represents the path duration in a generic Nhop path when nodes follow the Modified Random WayPoint.

\section{RESULTS}

To verify the correctness of the abovementioned analytical results, the software module developed for link duration was extended in order to compute all routes that the nodes could establish based on a connectivity graph. Once the computation of link duration is completed, it is straightforward to extend it in order to calculate the duration of a sequence of links, i.e. a path.

The Figure 2 compares the analytical results obtained from the analytical model of section 4 to those provided by the simulation of a representative scenario. Similar approximations are obtained when other conditions are configured. 


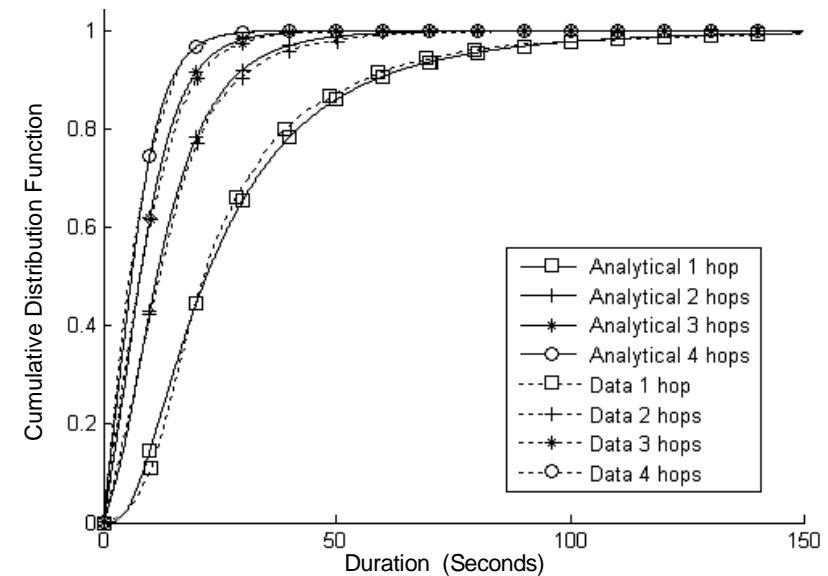

Figure 2. Comparison of CDF for paths of several hops. Scenario parameters are: Uniform speed, Vmax $=10 \mathrm{~m} / \mathrm{s}, V \min =1 \mathrm{~m} / \mathrm{s}$, Pause $=$ $0 \mathrm{~s}$, Tx range $=100 \mathrm{~m}$, Nodes $=15$, Area $=1500 \times 300 \mathrm{~m}^{2}$

As shown, simulation data closely follow analytical results. Therefore the analytical model approximates accurately the path duration. As exposed in Figure 2, path duration decreases when the number of hops that it contains increases.

\section{CONCLUSIONS}

In this paper authors propose an analytical model for link duration when nodes follow the extendedly used Random WayPoint. This model has been verified by means of simulations across 50 scenarios enclosing different mobility and transmission conditions. From the link model, path duration model is constructed and checked again via simulations. Both ns and Matlab simulations were run. The simulation results are well approximated by the analytical equations.

\section{REFERENCES}

[1] C. Perkins, E. Belding-Royer and S. Das, "Ad hoc OnDemand Distance Vector (AODV) Routing”, IETF RFC 3561, July 2003.

[2] D.B. Johnson, D.A. Maltz and Y.C. Hun, "The Dynamic Source Routing Protocol for Mobile Ad Hoc Networks (DSR)”, IETF Internet Draft, Work in progress, July 2004.

[3] I. Chakeres, C. Perkins, "Dynamic MANET On-demand (DYMO) Routing”, IETF Internet Draft, Work in progress, March 2006.

[4] D. Turgut, S.K. Das, M. Chatterjee, "Longevity of Routes in Mobile Ad hoc Networks", Proc. of IEEE Vehicular Technology Conference VTC 2001, Spring 2001, 2833-2837 vol.4, Rhodes (Greece)

[5] F. Bai, N. Sadagopan , B. Krishnamachari, A. Helmy, "Modelling Path Duration Distributions in MANETS and Their Impact on Reactive Routing Protocols", IEEE J. on Selected Areas in Communications, v. 22, 1357- 1373, no. 7, pp. 1357- 1373, September 2004.

[6] Y. Han, R. La, A. Makowski and S. Lee, "Distribution of path durations in mobile ad hoc networks - Palm's Theorem to the rescue". To appear in Computer Network.
[7] S. Jiang, D. He and J. Rao, "A Prediction-based Link Availability Estimation for Mobile Ad Hoc Networks", IEEE/ACM Trans. on Networking (TON), Issue 6, pp.1302 - 1312, December 2005

[8] J. Brosch, D. A. Maltz, D. B. Johnson, Y.C. Hu, and J. Jetcheva, "A Performance Comparison of Multi-Hop Wireless Ad Hoc Network Routing Protocols", Proc. of the $4^{\text {th }}$ Annual ACM/IEEE International Conf. on Mobile Computing and Networking, pp. 85-97, Dallas (USA), Oct. 1998

[9] S. Arbindi, K. Namuduri and R. Pendse, "Statistical Estimation of Route Expiry Times in On-demand Ad hoc Routing Protocols", Proc. of the Second IEEE International Conference on Mobile Ad Hoc and Sensor Systems (MASS 05), Washington (USA), November 2005.

[10] S. Cho and J.P. Hayes, "Impact of Mobility on Connection Stability in Ad Hoc Networks", Proc. of IEEE Communication Society, WCNC 2005, vol. 3, pp.1650-1656, New Orleans (USA).

[11] Y.C. Tseng, Y.F. Li, and Y.C. Chang, "On Route Lifetime in Multihop Mobile Ad Hoc Networks", IEEE Trans. on Mobile Computing, Vol. 2, No. 4, pp. 366-376, October 2003.

[12] D. Yu, H. Li and I. Gruber, "Path Availability in Ad Hoc Network", in Telecommunications, ICT 10th International Conf. vol.1, pp.383-387, March 2003.

[13] T. Camp, J. Boleng and V. Davies, "A Survey of Mobility Models for Ad Hoc Network Research", Wireless Communications \& Mobile Computing (WCMC): Special issue on Mobile Ad Hoc Networking: Research, Trends and Applications, April 2002.

[14] J. Boleng, W. Navidi and T. Camp, "Metrics to Enable Adaptive Protocols for Mobile Ad hoc Networks', Proc. of the International Conference on Wireless Networks (ICWN'02), pp.293-298, 2002.

[15] A.B. McDonald and T.F. Znati, "A path availability model for wireless ad-hoc networks", Proc. of IEEE WCNC, pp. 35-40, September 1999.

[16] P.Samar and S.B. Wicker, "On the Behavior of Communication Links of a Node in Multi-Hop Mobile Environment", In Proc. of the 5th ACM international symposium on Mobile ad hoc networking and computing, pp.145-156, 2004.

[17] J.Yoon, M. Liu, and B. Noble, "Random waypoint considered harmful”, Proc. of INFOCOM'03, pp. 1312-1321, San Francisco. April 2003.

[18] M. Gerharz, C. de Waal, P. Martín and P. James, "Strategies for Finding Stable Paths in Mobile Wireless Ad Hoc Networks", Proc. of the 28th IEEE Conference on Local Computer Networks (LCN), pp.130-139, Köngiswinter (Germany), October 2003.

[19] T. Dimitar, F. Sonja,C. Bekim, G. Aksenti, "Link Realiability analysis in ad hoc networks", Proc. of XII Telekomunikacioni forum TELFOR 2004.

[20] http://www.mathworks.com/

[21] http://www.isi.edu/nsnam/ns 